\title{
Pulse wave velocity and carotid atherosclerosis in White and Latino patients with hypertension
}

Mori J Krantz ${ }^{1,2^{*}}$, Carlin S Long ${ }^{1,2}$, Patrick Hosokawa ${ }^{3}$, Elhum Karimkhani ${ }^{1}$, Miriam Dickinson ${ }^{3}$, Raymond O Estacio ${ }^{1,2}$, Frederick A Masoudi ${ }^{1,2}$ and Edward P Havranek ${ }^{1,2}$

\begin{abstract}
Background: Preventive cardiology has expanded beyond coronary heart disease towards prevention of a broader spectrum of cardiovascular diseases. Ethnic minorities are at proportionately greater risk for developing extracoronary vascular disease including heart failure and cerebrovascular disease.

Methods: We performed a cross sectional study of Latino and White hypertension patients in a safety-net healthcare system. Framingham risk factors, markers of inflammation (hsCRP, LPpLA2), arterial stiffness (Pulse wave velocity, augmentation index, and central aortic pressure), and endothelial function (brachial artery flow-mediated dilatation) were measured. Univariate and multivariable associations between these parameters and an index of extracoronary atherosclerosis (carotid intima media thickness) was performed.

Results: Among 177 subjects, mean age was 62 years, 67\% were female, and 67\% were Latino. In univariate analysis, markers associated with carotid intima media thickness (IMT) at $p<0.25$ included pulse wave velocity (PWV), augmentation index (Alx), central aortic pressure (CAP), and LPPLA 2 activity rank. However, Alx, cAP, and LPPLA2 activity were not significantly associated with carotid IMT after adjusting for Framingham risk factors (all $p>$.10). Only PWV retained a significant association with carotid IMT independent of the Framingham general risk profile parameters $(p=.016)$. No statistically significant interactions between Framingham and other independent variables with ethnicity (all $p>.05$ ) were observed.
\end{abstract}

Conclusion: In this safety net cohort, PWV is a potentially useful adjunctive atherosclerotic risk marker independent of traditional risk factors and irrespective of ethnicity.

Keywords: Pulse wave velocity hypertension, atherosclerosis, carotid intima media thickness, Latino, inflammatory markers, augmentation index, central aortic pressure, C-reactive protein

\section{Background}

An emerging concept in cardiovascular disease (CVD) prevention is a shift in focus from predicting isolated coronary events to broader prediction of any CVD event. This has led to derivation of a new Framingham CVD risk profile [1] beyond the traditional score for predicting 10-year coronary disease events [2]. To illustrate this, data from the National Health and Nutrition Examination Surveys compared the two instruments among US men and women and found that just $3 \%$ of individuals were at a high $(>20 \%)$ predicted coronary disease risk, whereas $18 \%$ were at high CVD risk when CVD death,

\footnotetext{
* Correspondence: mkrantz@dhha.org

${ }^{1}$ Cardiology Division, Denver Health Medical Center, Denver, USA

Full list of author information is available at the end of the article
}

myocardial infarction, angina, stroke, transient ischemic attack, peripheral arterial disease, and heart failure were encompassed [3]. This broader focus on predicting CVD risk might especially benefit ethnic minorities given higher prevalence rates of non-coronary vascular disease [4]. Although the prevalence of coronary disease is higher for Whites versus minorities, total CVD rates are higher among minorities, largely attributable to a higher occurrence of stroke and heart failure [4].

At the same time, considerable efforts have been made to improve the discriminative capacity of risk prediction tools by adding new risk factors to the models. Markers of inflammation, particularly high-sensitivity C-reactive protein (hsCRP), and measures of vascular function have received attention. The role of emerging markers has
C Biomed Central 
not been well explored for prediction of CVD beyond coronary disease, and data are more limited among minority populations. For instance, just $1 \%$ of subjects in both derivation and validation cohorts of a study assessing the additional predictive value of hsCRP were Latino [5]. Although a greater prevalence of arterial stiffness has been demonstrated in African Americans [6], little is known about vascular function among Latinos, the largest and fastest growing minority population in the United States [7]. Given this background, we evaluated inflammatory and vascular function markers in a safety-net population with chronic hypertension. We hypothesized that regardless of ethnicity, pulse wave velocity (PWV) would be associated with pre-clinical carotid atherosclerosis beyond traditional risk factors.

\section{Methods}

\section{Subject characteristics}

The study sample consisted of 177 subjects recruited from an electronic registry of hypertension patients created as part of the Latinos Using Cardio Actions to Reduce Risk (LUCHAR) program [8]. Patients in the registry were regular patients at Denver Health, an integrated urban safety net health system [9]. Data from the registry were supplemented with chart review and patient self-report during an enrollment interview. Patients were eligible for the current study if they were $\geq 18$ years of age, of either Latino or non-Latino White ethnicity, actively receiving antihypertensive medication, and had an established diagnosis of chronic hypertension. In addition, subjects had at least one other CVD risk factor including diabetes, dyslipidemia, obesity, chronic kidney disease, microalbuminuria, current smoking, or age $>55$ for men or $>65$ for women. All participants provided written informed consent in order to participate in the study. Patients were evaluated consecutively during the enrollment period and excluded if they had pre-existing CVD defined as myocardial infarction, prior percutaneous or surgical coronary revascularization, stroke, cerebrovascular revascularization, or peripheral arterial disease. Additional exclusions were valvular heart disease, end-stage renal disease, inflammatory disease, active substance abuse, or projected life expectancy $<12$ months. Non-invasive vascular structure and function markers as well as inflammatory biomarkers were obtained on a single day. The Colorado Multiple Institutional Review Board approved the study protocol.

\section{Inflammatory markers}

A venous plasma sample was obtained from all subjects. Patients were asked to fast and refrain from smoking from the night before their appointment. We chose two markers of systemic inflammation, high sensitivity
C-reactive protein (hsCRP) because it has been studied extensively as a risk factor ${ }^{5}$ and lipoprotein-associated phospholipase A2 (Lp-PLA2) given its purported specificity for inflammation localized to atherosclerotic plaque including carotid arteries [10-13]. Levels of Lp-PLA2 mass and activity were measured (PLAC; diaDexus Inc, South San Francisco, CA) by a dual monoclonal antibody immunoassay. ${ }^{13}$ Samples were assayed for hsCRP with a high-sensitivity assay (hsCRP Flex ${ }^{\circledR}$, Dimension Vista). This assay was validated against the Roche Hitachi Modular assay and found to have acceptable precision and reproducibility [14].

\section{Vascular function measures}

All measurements were performed in a quiet room with controlled ambient temperature. Blood pressure was measured in duplicate in the supine position using the non-dominant arm in all subjects. Brachial-ankle pulse wave velocity (PWV) was derived from the pulse transit time between and the estimated path length between proximal and distal arterial sites expressed as $\mathrm{cm} / \mathrm{sec}$. Applanation tonometry (HEM-9000AI Omron Healthcare, Bannockburn, Illinois) was performed at the radial artery to derive the augmentation index (AIx) and central aortic pressure (cAP). AIx was calculated as the difference between the first (ejection) and second (reflected) peaks of the arterial waveform, expressed as a percentage of the pulse pressure, where higher AIx values reflect greater vascular stiffness.

One ultrasonographer assessed flow-mediated dilation (FMD) of the brachial artery in the non-dominant arm, which was immobilized and scanned above the antecubital fossa using a 5-12 MHz linear array transducer (Phillips Medical Systems model iE33, Bothell, WA). Arterial images were obtained before (baseline) and after (maximal) inflation of a cuff at pressures $>40 \mathrm{~mm} \mathrm{Hg}$ above systolic blood pressure for five minutes. Longitudinal images were scanned and captured (in $\mathrm{mm}$ ) at end diastole. FMD was defined as [maximal - baseline brachial artery diameter] divided by baseline brachial artery diameter $\times 100$ and expressed as percentages.

\section{Carotid intima-media thickness}

Measures of maximal carotid IMT were obtained in the supine position by a single ultrasonographer. Longitudinal B-mode ultrasound images were obtained among subjects with the head turned 45 degrees from the area scanned. Gain settings were optimized to acquire far wall arterial images and limit echogenicity of the lumen. A linear array probe (Phillips Sonos 5500, Netherlands) was used for all image acquisition. The sonographer obtained 3 longitudinal views of both internal carotid arteries for a total of 6 IMT images per subject as previously described [15]. The internal carotid artery was 
defined to include the bulb and the initial $10 \mathrm{~mm}$ of vessel distal to separation of external from internal arteries. High resolution images were stored digitally, and read off-line by trained interpreters blinded to clinical characteristics of study participants. Near and fall wall thickness were calculated as the maximum distance between the lines.

\section{Statistical Analysis}

Mean and standard deviations for all normally distributed variables were calculated. Pulse wave velocity was normalized after transformation by the relationship Pinverse $=-(1 / \mathrm{PWV})-0.0006) / 0.00011)[16]$. Carotid IMT was the primary outcome variable. Pearson correlation coefficients or Student's t-test were used to assess univariate relationships between carotid IMT and candidate independent variables. Multivariate linear regression models were used to assess the association between carotid IMT and the inflammatory, vascular stiffness, and endothelial function variables after adjusting for variables used in the Framingham general risk profile including age, gender, systolic blood pressure, anti-hypertensive medication use, total and HDL-cholesterol, diabetes, and smoking status. Because all subjects were receiving antihypertensive medications, this variable was not considered further. P-values $<0.05$ were considered statistically significant. Receiver operator characteristic curves to predict high carotid IMT as a dichotomous variable were also constructed for Framingham risk score, PWV, and AIx. We tested interactions with each independent variable and ethnicity in another set of models to test the hypothesis that the relationship between the outcome variables and the independent variables would be similar between Latino and non-Latino white subgroups. Following data quality assurance measures, SAS Version 8.0 (Cary, NC) was used for all statistical analyses.

\section{Results}

Baseline characteristics of the sample are shown in Table 1. The mean age was 62 years, two thirds were female and two-thirds were Latino. Nearly half had diabetes. Consistent with a sample drawn from a safety net institution, nearly half had not completed high school and over $80 \%$ were unemployed or disabled. Mean LpPLA2 was $173 \pm 59 \mathrm{ng} / \mathrm{ml}$. In contrast with hsCRP, only $21 \%$ of subjects would be classified as high-risk, using the LpPLA2 cut-point of $>200 \mathrm{ng} / \mathrm{ml}$ [17]. Mean hsCRP was $5.25 \pm 6.65 \mathrm{mg} / \mathrm{L}$ and over $63 \%$ of the cohort would be classified as high-risk using the recently established cut-point of $2 \mathrm{mg} / \mathrm{L}$ used in the JUPITER trial [18]. Univariate relationships between independent variables and carotid IMT are shown in Table 2 . The inflammatory, and vascular function variables associated with cIMT at $\mathrm{p}<0.25$ on univariate analysis were PWV,
Table 1 Patient Characteristics $(n=177)$

\begin{tabular}{|c|c|}
\hline Age, years & $61.5(9.7)$ \\
\hline Female gender & $66.7 \%$ \\
\hline \multicolumn{2}{|l|}{ Ethnicity } \\
\hline Hispanic & $66.7 \%$ \\
\hline Non-Hispanic White & $33.3 \%$ \\
\hline \multicolumn{2}{|l|}{ Education } \\
\hline College graduate & $8.5 \%$ \\
\hline Higher education & $19.3 \%$ \\
\hline High school graduate & $19.8 \%$ \\
\hline Some high school & $21.5 \%$ \\
\hline No high school & $31.1 \%$ \\
\hline \multicolumn{2}{|l|}{ Employment Status } \\
\hline Employed & $19.2 \%$ \\
\hline Unemployed & $46.9 \%$ \\
\hline Disabled & $33.9 \%$ \\
\hline Body mass index, $\mathrm{kg} / \mathrm{m}^{2}$ & $33.0(6.7)$ \\
\hline Blood pressure, $\mathrm{mmHg}$ & $\begin{array}{c}142.2 \pm 22.1 / 81.7 \\
\pm 12.3\end{array}$ \\
\hline Total cholesterol, mg/dl & $181.5(39.6)$ \\
\hline Triglycerides, mg/dl & $170.9(105.2)$ \\
\hline HDL cholesterol, mg/d & $51.0(12.8)$ \\
\hline LDL-cholesterol, mg/dl & $96.8(33.3)$ \\
\hline Diabetes Mellitus & $49.2 \%$ \\
\hline Current Smoker & $22.0 \%$ \\
\hline Framingham risk score* & $11.6(8.0), 1-53$ \\
\hline $\operatorname{LpPLA2}^{\dagger}$ mass, $\mathrm{ng} / \mathrm{mL}$ & $173.5(59.0)$ \\
\hline LpPLA2 activity, $\mathrm{nmol} / \mathrm{min} / \mathrm{mL}$ & $134.8(34.3)$ \\
\hline Central aortic pressure, $\mathrm{mm} \mathrm{Hg}$ & $59.5(18.1)$ \\
\hline Augmentation index & $86.3 \%(12.7)$ \\
\hline Pulse wave velocity, $\mathrm{cm} / \mathrm{sec}$ & $1723.3(349)$ \\
\hline Flow-mediated dilation & $10.5 \%(8.1)$ \\
\hline Carotid intima-media thickness, mm & $0.95(0.22)$ \\
\hline Use of Hypertension Medication & $90.4 \%$ \\
\hline $\begin{array}{l}\text { Use of Hydroxy-3-methylglutaryl Co-enzyme A } \\
\text { reductase inhibitors (statins) }\end{array}$ & $59.9 \%$ \\
\hline
\end{tabular}

Values expressed as mean + standard deviation (SD) or as percentages (\%). hsCRP $=$ high sensitivity C-reactive protein. ${ }^{*}$ mean (SD) and range ${ }^{\dagger}$ LpPLA2 = Lipoprotein-associated phospholipase A2.

AIx, cAP and LpPLA 2 activity rank. PWV had the strongest linear relationship $(\mathrm{r}=+0.39, \mathrm{p}<0.0001)$ with carotid IMT (Figure 1). For high carotid IMT (>0.8 mm) $\mathrm{C}$-statistics assessing discrimination for PWV, AIx and Framingham risk score (adjusting for age, gender, systolic blood pressure, and ethnicity) were $0.72,0.63$, and 0.51 respectively. Results of the multiple linear regression analyses with standardised coefficients are shown in Table 3. After adjusting for Framingham risk factors, AIx, cAP, and LpPLA2 activity were no longer significantly associated with carotid IMT (all p > .10). Only PWV remained associated with carotid IMT independent of the Framingham general risk profile parameters $(\mathrm{p}=.016)$. Additional analyses indicated no statistically 
Table 2 Univariate relationships with Carotid Intima Media Thickness

\begin{tabular}{lcc}
\hline $\mathbf{N}=177$ & Carotid intima-media thickness \\
\hline & Correlation coefficient & $\mathbf{P}$ \\
\hline Age (years) & 0.369 & $<.0001$ \\
Systolic blood pressure (mm Hg) & 0.207 & 0.006 \\
Total cholesterol (mg/dL) & 0.054 & 0.476 \\
HDL cholesterol (mg/dL) & 0.096 & 0.210 \\
Pulse wave velocity (cm/s) & 0.389 & $<.0001$ \\
Augmentation Index (\%) & 0.123 & 0.130 \\
Central aortic pressure (mm Hg) & 0.254 & 0.005 \\
hsCRP (mg/L) & -0.004 & 0.959 \\
LPPLA2 Mass rank & 0.034 & 0.653 \\
LPPLA2 Activity rank & 0.110 & 0.149 \\
Flow-mediated dilation (\%) & -0.087 & 0.270 \\
Gender & -0.170 & 0.007 \\
Current Smoker & 0.023 & 0.713 \\
Diabetes & -0.046 & 0.461 \\
\hline hsCRP
\end{tabular}

hsCRP = high sensitivity C-reactive protein. LpPLA2 = Lipoprotein-associated phospholipase A2. $\mathrm{HDL}=$ high density lipoprotein.

significant interactions between the Framingham risk factors and other independent variables and ethnicity (all $\mathrm{p}>$.05) when predicting carotid IMT. For the interaction between PWV and ethnicity when predicting carotid IMT, the p-value was 0.518 .

\section{Discussion}

In this cross-sectional study of a mixed-ethnicity population, PWV, a measure of arterial stiffness, was associated with preclinical carotid atherosclerosis independent of Framingham risk factors. To our knowledge, this is the first study to demonstrate that PWV is associated with preclinical atherosclerosis among a Latinopredominant population. Findings were similar in both Latino and non-Latino White hypertensive subjects, suggesting that measures of arterial stiffness could play a role in CVD risk stratification within the growing US safety net population. Moreover, two other arterial stiffness measures, two inflammatory markers, and a traditional measure of endothelial function were not associated with carotid intima media thickness.

Overall, most studies to date included patients with a wide range of ages and underlying conditions but a limited range of race and ethnicity. In particular, none included substantial numbers of Latinos. Moreover, very few studies have compared the relative value of arterial stiffness, inflammatory markers, and endothelial function as correlates of CVD beyond isolated coronary disease events. An epidemiological study of 2,191 elderly (age at baseline 70-79) African American and White subjects, assessed the predictive value of PWV, ankle brachial index (ABI), and inflammatory markers (hsCRP,

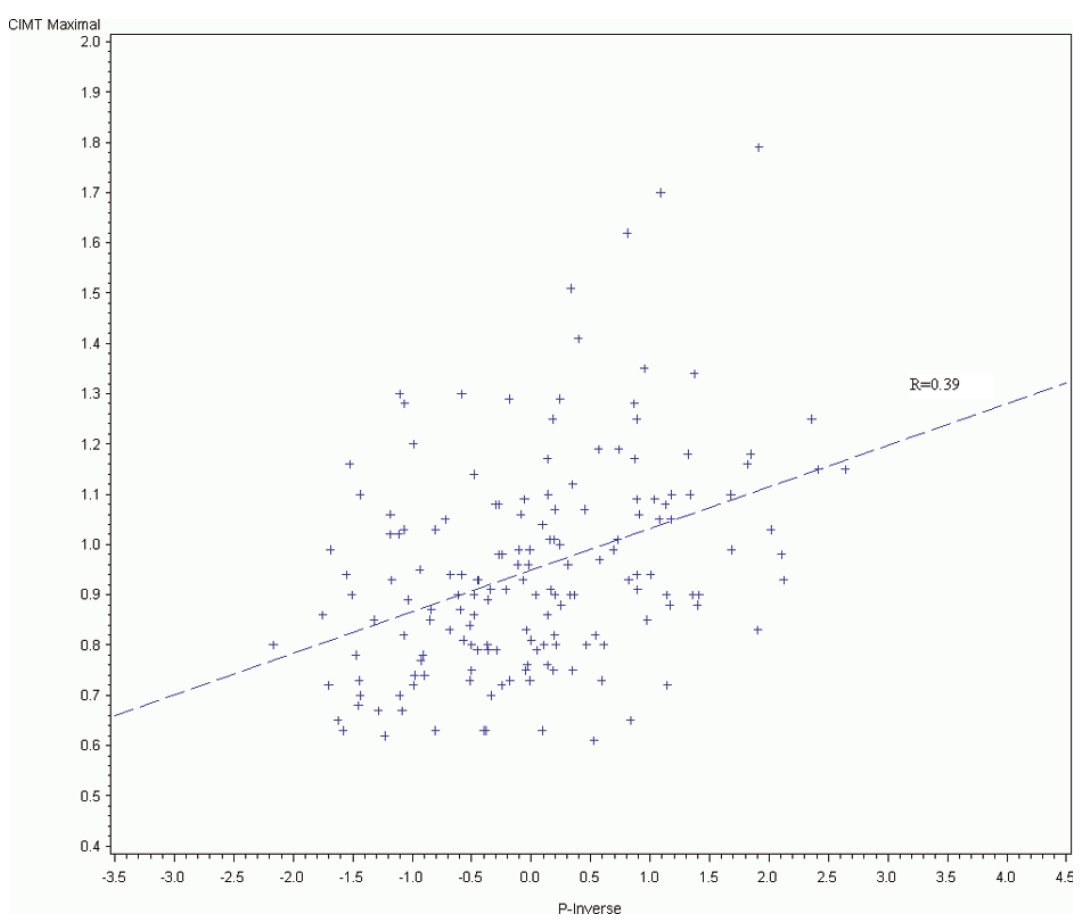

Figure 1 Relationship of pulse wave velocity to carotid intima media thickness. The relationship between the inverse of the brachial-ankle pulse wave velocity (PWV) in $\mathrm{cm} / \mathrm{second}$ and the carotid intima media thickness in mm. P-inverse is - (1/PWV -0.0006$) / 0.00011)$. The resulting Z (P-inverse) quantity has a mean of 0 and an SD of 1 . The negative sign is used to ensure that correlations with PWV retain the same direction when compared to P-inverse. 
Table 3 Multivariate relationships with carotid intima-media thickness $(n=177)$

\begin{tabular}{|c|c|c|c|c|c|c|c|c|c|c|}
\hline & \multicolumn{2}{|c|}{$\begin{array}{l}\text { Framingham factors } \\
\text { alone }\end{array}$} & \multicolumn{2}{|c|}{$\begin{array}{c}\text { Framingham factors } \\
+ \text { PWV }\end{array}$} & \multicolumn{2}{|c|}{$\begin{array}{l}\text { Framingham factors } \\
+ \text { Alx }\end{array}$} & \multicolumn{2}{|c|}{$\begin{array}{l}\text { Framingham factors } \\
+ \text { CAP }\end{array}$} & \multicolumn{2}{|c|}{$\begin{array}{c}\text { Framingham factors } \\
+\mathrm{LpPLA}_{2}\end{array}$} \\
\hline & estimate & $\mathbf{P}$ & estimate & $\mathbf{P}$ & estimate & $\mathbf{P}$ & estimate & $\mathbf{P}$ & estimate & $\mathbf{P}$ \\
\hline Age & 0.074 & $<.0001$ & 0.045 & 0.016 & 0.073 & $<.0001$ & 0.068 & 0.004 & 0.075 & $<.0001$ \\
\hline Female Gender & -0.111 & 0.002 & -0.113 & 0.001 & -0.127 & 0.002 & -0.144 & 0.001 & -0.113 & 0.002 \\
\hline Systolic BP & 0.025 & 0.119 & 0.017 & 0.323 & 0.012 & 0.507 & 0.008 & 0.819 & 0.025 & 0.115 \\
\hline Total Cholesterol & 0.014 & 0.397 & 0.003 & 0.862 & 0.005 & 0.772 & 0.005 & 0.809 & 0.018 & 0.296 \\
\hline HDL Cholesterol & 0.018 & 0.303 & 0.022 & 0.205 & 0.024 & 0.201 & 0.024 & 0.245 & 0.017 & 0.322 \\
\hline Current Smoker & 0.011 & 0.782 & -0.003 & 0.937 & 0.023 & 0.596 & 0.007 & 0.893 & 0.018 & 0.659 \\
\hline Diabetes & -0.008 & 0.796 & -0.010 & 0.745 & 0.000 & 0.994 & -0.025 & 0.562 & -0.010 & 0.763 \\
\hline Pulse wave velocity & & & 0.049 & 0.016 & & & & & & \\
\hline Augmentation Index & & & & & 0.021 & 0.295 & & & & \\
\hline Central Aortic Pressure & & & & & & & 0.034 & 0.384 & & \\
\hline $\mathrm{LpPLA}_{2}$ & & & & & & & & & -0.013 & 0.441 \\
\hline
\end{tabular}

interleukin-6, and TNF- $\alpha$ ) to Framingham risk factors [19]. The outcome variable was limited to coronary disease events. PWV, ABI, and interleukin- 6 were independent predictors, and the model with the best discriminative capacity added both ABI and interleukin- 6 to Framingham factors. These results differ from our finding that PWV was associated with preclinical cardiovascular disease whereas inflammatory markers were not. By contrast, a study of 558 patients with hypertension and without baseline CVD, added PWV and FMD in Cox multi-variable models that had occurrence of CVD events including stroke as the outcome variable [20]. PWV was related to the occurrence of CVD events while FMD was not, consistent with the results of the current study in a similar hypertensive cohort. Our findings are also consistent with the current consensus opinion that PWV is the gold standard method for assessing arterial stiffness [21].

Our results extend to a multi-ethnic population findings of an association between arterial stiffness and cardiovascular outcomes. In a recent meta-analysis, Vlachopoulos et al [22] combined results of 17 longitudinal studies and found a relative risk of 1.47 for cardiovascular events associated with each standard deviation increase in PWV. These 17 studies varied in the degree to which they accounted for Framingham risk factors and in the degree to which they considered a full range of non-coronary CVD outcomes. The most comprehensive study was performed by Mitchell et al [16], and was consistent with our study in that PWV, but not AIx or cAP, was a significant predictor of CVD events independent of the Framingham general risk profile parameters.

A number of factors should be considered in interpreting the results of the current investigation. This was a cross-sectional study and was not powered to predict CVD events. With a larger sample size, additional vascular function variables could have reached statistical significance. Relative to PWV, however, cAP and AIx are less direct measures of arterial stiffness, more sensitive to differences in heart rate and body size, and may be more technically challenging to measure thereby limiting their utility in risk stratification. The suggestion that $\mathrm{LPPLA}_{2}$ may have greater predictive value than hsCRP in high-risk populations deserves further exploration since hsCRP values were uniformly elevated in this safety net population. The absence of even a univariate association between hsCRP and carotid IMT in the current study is informative given the widespread use of this marker in clinical practice. A recent guideline affirms that measurement of hsCRP is not recommended for risk stratification in asymptomatic higherrisk adults, nor among older low-risk adults [23]. Confirmatory longitudinal studies comparing vascular function and inflammatory markers as predictors of CVD in samples with mixed race and ethnicity are needed to better assess the strength and clinical implications of this association. In addition, the prognostic significance of brachial-ankle PWV is less established compared with carotid-femoral PWV. However, brachial-ankle PWV, is more easily obtained and has been shown to be highly correlated $(\mathrm{r}=0.76, \mathrm{p}<$ 0.0001 ) with carotid-femoral PWV values among hypertensive patients [24]. Because of its strong relationship with aortic pulse wave velocity, brachial-ankle PWV measurements offers both practical and theoretic advantages over the more frequently used carotid-femoral method [25], though confirmatory studies with carotidfemoral PWV are warranted. One strength of the current analysis is its focus on a traditionally under-studied safety net population. Evaluating sociodemographically vulnerable subjects serves to broaden the applicability of research findings to those individuals typically excluded 
from research trials; in our study, the majority of participants were women and Latinos in marked contrast to typical cardiovascular trials [26].

\section{Conclusions}

Despite its strong association with cardiovascular mortality among hypertensive patients [27] and endorsement as a gold standard for vascular stiffness, the value of PWV as a therapeutic target remains uncertain. Nonetheless, our study demonstrates that PWV, in contrast to inflammatory biomarkers, has predictive value for preclinical atherosclerosis beyond the Framingham risk profile among subjects of mixed ethnicity. Given ongoing concerns about the inaccuracy of the Framingham risk score in racial/ethnic minorities [28] and the importance of considering overall CVD risk in such individuals, PWV warrants further evaluation in both epidemiologic and interventional studies.

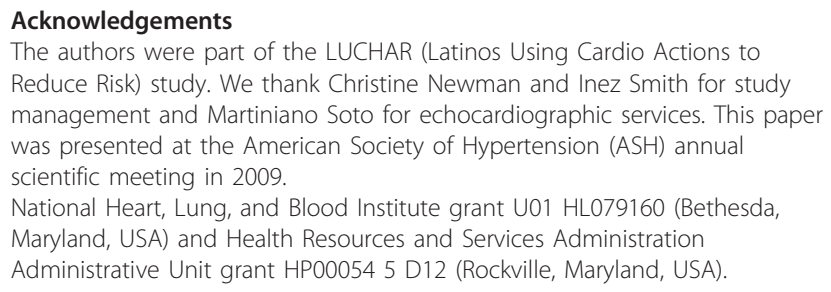

\section{Author details}

${ }^{1}$ Cardiology Division, Denver Health Medical Center, Denver, USA

2Department of Medicine, University of Colorado School of Medicine, Aurora, USA. ${ }^{3}$ Department of Family Medicine, University of Colorado School of Medicine, Aurora, USA.

\begin{abstract}
Authors' contributions
MJK was the lead on non-invasive hemodynamic data and carried out primary drafting of the paper and study design, CSL led the biomarker analysis and contributed to study design and substantive edits, $\mathrm{PH}$ performed all statistical analyses, tables' and figures and was involved in quality control. EK led the acquisition and interpretation of data and made substantive intellectual contributions. MD was the senior statistician and provided critical content review. ROE made substantial contributions to conception and design. FAM was responsible for IMT measurements and interpretation as well as key analysis. EPH conceived of and designed the overall study and contributed substantial time to analysis and framing the paper. All authors read and approved the final manuscript.
\end{abstract}

\section{Competing interests}

The authors declare that they have no competing interests.

Received: 7 November 2010 Accepted: 11 April 2011

Published: 11 April 2011

\section{References}

1. D'Agostino RB Sr, Vasan RS, Pencina MJ, Wolf PA, Cobain M, Massaro JM, Kannel WB: General cardiovascular risk profile for use in primary care: the Framingham Heart Study. Circulation 2008, 117:743-753.

2. Wilson PW, D'Agostino RB Sr, Levy D, Belanger AM, Silbershatz H, Kannel WB: Prediction of coronary heart disease using risk factor categories. Circulation 1998, 97:1837-1847.

3. Marma AK, Ning H, Lloyd-Jones DM: Impact of a change to global cardiovascular risk estimation in lipid-lowering guidelines: Finding from the National Health and Nutrition Examination Survey (NHANES) 20012006. Circulation 2009, 120:5424.

4. Lloyd-Jones D, Adams RJ, Brown TM, on behalf of the American Heart Association Statistics Committee and Stroke Statistics Subcommittee, et al: Heart disease and stroke statistics-2010 update: a report from the American Heart Association. Circulation 2010, 121:e1-e170.

5. Ridker PM, Buring JE, Rifai N, Cook NR: Development and validation of improved algorithms for the assessment of global cardiovascular risk in women: the Reynolds Risk Score. JAMA 2007, 297:611-619.

6. Heffernan KS, Fahs CA, Iwamoto GA, Jae SY, Wilund KR, Woods JA, Fernhall B: Resistance exercise training reduces central blood pressure and improves microvascular function in Afrincan American and white men. Atherosclerosis 2009, 207:220-226.

7. [http://www.censusscope.org/us/map_hispanicpop.html], Accessed 25 December 2009

8. Hanratty R, Estacio RO, Dickinson LM, Chandramouli V, Steiner JF, Havranek EP, Latino Using Cardio Health Actions to Reduce Risk study investigators: Testing electronic algorithms to create disease registries in a safety net system. J Health Care Poor Underserved 2008, 19:452-465.

9. Gabow P, Eisert S, Wright R: Denver Health: a model for the integration of a public hospital and community health centers. Ann Int Med 2003, 138:143-149.

10. Lavi S, McConnell JP, Rihal CS, Prasad A, Mathew V, Lerman LO, Lerman A: Local production of lipoprotein-associated phospholipase A2 and lysophosphatidylcholine in the coronary circulation: association with early coronary atherosclerosis and endothelial dysfunction in humans. Circulation 2007, 115:2715-2721.

11. Kolodgie FD, Burke AP, Skorija KS, Ladich E, Kutys R, Makuria AT, Virmani R: Lipoprotein-associated phospholipase A2 protein expression in the natural progression of human coronary atherosclerosis. Arterioscler Thromb Vasc Biol 2006, 26:2523-2529.

12. Mannheim D, Herrmann J, Versari D, Gossl M, Meyer FB, McConnell JP, et al: Enhanced expression of Lp-PLA2 and lysophosphatidylcholine in symptomatic carotid atherosclerotic plaque. Stroke 2008, 39:1448-1455.

13. Davidson MH, Corson MA, Alberts MJ, Anderson JL, Gorelick PB, Jones PH: Consensus panel recommendation for incorporating lipoproteinassociated phospholipase A2 testing into cardiovascular disease risk assessment guidelines. Am J Cardiol 2008, 101:51F-57F.

14. Clinical and Laboratory Standards Institute (CLSI): Methods comparison and bias estimation using patient samples; approved guideline-second edition. CLSI document EP9-A2 (ISBN 1-56238-472-4) CLSI, 940 West Valley Road, Suite 1400, Wayne PA 19087-1898 USA; 2002.

15. O'Leary DH, Polak JF, Wolfson SK Jr, et al: Use of sonography to evaluate carotid atherosclerosis in the elderly. The Cardiovascular Health Study. CHS Collaborative research Group. Stroke 1991, 22:1155-63.

16. Mitchell GF, Hwang SJ, Vasan RS, Larson MG, Pencina MJ, Hamburg NM, et al: Arterial stiffness and cardiovascular events: the Framingham Heart Study. Circulation 2010, 121:505-511.

17. Kardys I, Oei HHS, van der Meer IM, Horman A, Breteler MMB, Witteman JCM: Lipoprotein-associated phospholipase A2 and measures of extracoronary atherosclerosis: the Rotterdam study. Aterioscler Thromb Vasc Biol 2006, 26:631-636.

18. Ridker PM, Danielson E, Fonseca FA, Genest J, Gotto AM Jr, Kastelein JJ, JUPITER Study Group, et al: Rosuvastatin to prevent vascular events in men and women with elevated C-reactive protein. N Engl J Med 2008, 359:2195-2207.

19. Rodondi N, Marques-Vidal P, Butler J, Sutton-Tyrrel K, Comuz J, Satterfield S, Harris T, Bauer DC, Ferrucci L, Vittinghoff E, Newman AB: Markers of atherosclerosis and inflammation for prediction of coronary heart disease in older adults. Am J Epidemiol 2010, 171:540-549.

20. Terai M, Ohishi M, Ito N, Takagi T, Tatara Y, Kaibe M, et al: Comparison of arterial functional evaluations a a predictor cardiovascular events in hypertensive patients: the Non-invasive Atherosclerotic Evaluation in Hypertension (NOAH) study. Hypertens Res 2008, 31:1135-1145.

21. Laurent S, Cockcroft J, Van Bortel L, Boutouyrie P, Giannattasio C, Havoz D, et al: Expert consensus document on arterial stiffness: methodological issues and clinical applications. Eur Heart J 2006, 27:2588-2605.

22. Vlachopoulos C, Aznaouridis K, Stefanidis C: Prediction of cardiovascular events and all-cause mortality with arterial stiffness: A systematic review and meta-analysis. J Am Coll Cardiol 2010, 55:1318-1327. 
23. Greenland P, Alpert JS, Beller GA, Benjamin EJ, Budoff MJ, Fayad ZA, Foster E, Hlatky MA, Hodgson JMcB, Kushner FG, Lauer MS, Shaw LJ, Smith SC Jr, Taylor AJ, Weintraub WS, Wenger NK: 2010 ACCF/AHA guideline for assessment of cardiovascular risk in asymptomatic adults: executive summary: a report of the American College of Cardiology Foundation/American Heart Association Task Force on Practice Guidelines. J Am Coll Cardiol 2010, 56:2182-99.

24. Munakata M, Ito N, Nunokawa T, Yoshinaga K: Utility of automated brachial ankle pulse wave velocity measurements in hypertensive patients. Am J Hypertens 2003, 16:653-657.

25. Sugawara J, Hayashi K, Yokio T, Cortez-Cooper MY, DeVan AE, Anton MA, Tanaka H: Brachial-ankle pulse wave velocity: an index of central arterial stiffness? J Hum Hypertens 2005, 19:401-406.

26. Hoel AW, Kayssi A, Brahmanandam S, Belkin M, Conte MS, Nguyen LL: Under-representation of women and ethnic minorities in vascular surgery randomized controlled trials. J Vasc Surg 2009, 50:349-54.

27. Laurent S, Boutouyre P, Asmar R, Gautier I, Laloux B, Guize L, et al: Aortic stiffness is an independent predictor of all-cause and cardiovascular disease mortality in hypertensive patients. Hypertension 2001, 37:1236-1241.

28. D'Agostion RB Sr, Grundy S, Sullivan LM, Wilson P, CHD Risk Prediction Group: Validation of the Framingham coronary heart disease risk prediction scores. Results of a multiple ethnic groups investigation. JAMA 2001, 286:180-187.

\section{Pre-publication history}

The pre-publication history for this paper can be accessed here: http://www.biomedcentral.com/1471-2261/11/15/prepub

doi:10.1186/1471-2261-11-15

Cite this article as: Krantz et al:: Pulse wave velocity and carotid atherosclerosis in White and Latino patients with hypertension. BMC Cardiovascular Disorders 2011 11:15.

\section{Submit your next manuscript to BioMed Central and take full advantage of:}

- Convenient online submission

- Thorough peer review

- No space constraints or color figure charges

- Immediate publication on acceptance

- Inclusion in PubMed, CAS, Scopus and Google Scholar

- Research which is freely available for redistribution

Submit your manuscript at www.biomedcentral.com/submit 